$41(2) \mid 2012$

Varia

\title{
Antigua fundición de plomo en el sitio inca de Curamba, departamento de Apurímac, Perú
}

Un ancien centre de fonderie de plomb, sur le site inca de Curamba, département d'Apurímac, Pérou

Ancient lead smelting at the Inca site of Curamba, Departament of Apurímac, Perú

William E. Brooks y Luisa Vetter Parodi

\section{OpenEdition}

Journals

Edición electrónica

URL: http://journals.openedition.org/bifea/567

DOI: $10.4000 /$ bifea.567

ISSN: 2076-5827

\section{Editor}

Institut Français d'Études Andines

\section{Edición impresa}

Fecha de publicación: 1 julio 2012

Paginación: 197-208

ISSN: 0303-7495

\section{Referencia electrónica}

William E. Brooks y Luisa Vetter Parodi, « Antigua fundición de plomo en el sitio inca de Curamba, departamento de Apurímac, Perú », Bulletin de l'Institut français d'études andines [En línea], 41 (2)| 2012, Publicado el 01 diciembre 2012, consultado el 27 noviembre 2020. URL : http:// journals.openedition.org/bifea/567 ; DOI : https://doi.org/10.4000/bifea.567

\section{(c) $(1) \odot$}

Les contenus du Bulletin de l'Institut français d'études andines sont mis à disposition selon les termes de la licence Creative Commons Attribution - Pas d'Utilisation Commerciale - Pas de Modification 4.0 International. 


\title{
Antigua fundición de plomo en el sitio inca de Curamba, departamento de Apurímac, Perú
}

\author{
William E. Brooks* \\ Luisa Vetter Parodi**
}

\begin{abstract}
Resumen
Desde el siglo XVI, el sitio inca de Curamba, ubicado en el departamento de Apurímac, en la sierra sur del Perú, ha sido interpretado como un centro metalúrgico para fundición de plata. Sin embargo, no hay restos u otros indicios del uso de huayras ni tocochimbos, que son estructuras usadas tradicionalmente para la antigua fundición de plata en los Andes. Los ensayos geoquímicos (Inductively Coupled Plasma o Plasma Acoplado Inductivamente) de la escoria extraída de los hornos de fundición en Curamba indican un bajo contenido de plata $(<0,3$ a $0,4 \mathrm{ppm})$, oro $(<2 \mathrm{ppm})$ y cobre (18 a $31 \mathrm{ppm})$, mientras que el contenido de plomo (155 a 234 ppm) y zinc (125 a 259 ppm) es alto. Otra evidencia de las ocurrencias de plomo-zinc es señalada por la presencia de arsénico, bario, hierro, manganeso y vanadio. Las rocas del sitio son calizas de grano fino, de color gris, con buzamiento al sur y pertenecen al grupo Pucará (Mesozoico). Unos pocos kilómetros al norte del sitio se encuentran rocas de la Formación Mitu, la base de Pucará, en tanto que a nivel regional hay ocurrencias de plomo-zinc asociadas con esa formación. Sin embargo, minerales de mena como la galena $(\mathrm{PbS})$ o la esfalerita [( $\mathrm{Zn}, \mathrm{Fe}) \mathrm{S}]$ no han sido encontrados en el sitio arqueológico. Usando la espectroscopía Mössbauer, se pudo determinar una temperatura de $800 \pm 50^{\circ} \mathrm{C}$ para los hornos de Curamba (Vetter et al., 2008). Esta temperatura es suficiente para fundir plomo $\left(\sim 300{ }^{\circ} \mathrm{C}\right)$, pero se requieren temperaturas más altas para fundir plata $\left(960{ }^{\circ} \mathrm{C}\right)$, oro $\left(1063{ }^{\circ} \mathrm{C}\right)$ o cobre $\left(1084{ }^{\circ} \mathrm{C}\right)$, mientras que las aleaciones de cobre-plata o cobre-oro requieren temperaturas de 780 a $880{ }^{\circ} \mathrm{C}$. Solo unos pocos artefactos precolombinos de plomo han sido descritos (Petersen, 2010 [1970]; Vetter \& Villacorta, 2001; Vetter, 2004; Velarde, 2010) y hay escasas referencias de sitios del antiguo Perú con evidencias de fundición de plomo (Wells, 1940; Howe \& Petersen, 1994).
\end{abstract}

Palabras clave: plomo, hornos, metalurgia, Plasma Acoplado Inductivamente

* Geólogo, Reston, VA 20191. E-mail: webgeology@aim.com

** Programa de Estudios Andinos, Pontificia Universidad Católica del Perú, Lima. Este trabajo es parte de mi tesis doctoral en Historia en el Programa de Estudios Andinos (PUCP). E-mail: luchivetter@ hotmail.com 


\title{
Un ancien centre de fonderie de plomb, sur le site inca de Curamba, département d'Apurímac, Pérou
}

\section{Résumé}

Depuis le 16e siècle, le site inca de Curamba (département d'Apurímac, sud du Pérou), est considéré comme un centre métallurgique ayant servi à fondre le minerai d'argent. Toutefois, on n'a jamais trouvé de restes, ou d'éléments permettant de confirmer l'utilisation de huayras ou de tocochimbos, traditionnellement utilisés pour la fonte de l'argent dans les Andes pré-hispaniques. Les essais géochimiques (Inductively Coupled Plasma) sur la scorie extraite des fours de la fonderie de Curamba indiquent une faible quantité d'argent ( $<0,3$ à 0,4 ppm), d'or ( $<2$ ppm), et de cuivre (18 à 31 ppm) tandis que le contenu de plomb (155 à 234 ppm) et de zinc (125 à 259 ppm) est élevé. Une autre preuve de la présence de l'alliage plomb-zinc est indiquée par la présence d'arsenic, de baryum, de fer, de manganèse et de vanadium. Les roches sont composées de calcaire fin, de couleur grise, avec une inclinaison vers le sud, appartenant au groupe Pucará (Mesosoique). À quelques kilomètres au nord du site on trouve des roches de la Formation Mitu, la base de Pucará, tandis que sur le plan régional, on trouve la présence de l'alliage plomb-zinc, associé à cette formation. Cependant, des minerais comme la galène $[\mathrm{PbS}]$ ou la sphalerite $[(\mathrm{Zn}, \mathrm{Fe}) \mathrm{S}]$ sont absents du site archéologique. L'utilisation de la spectroscopie de Mössbauer a permis de mettre en évidence une température de $800 \pm 50^{\circ} \mathrm{C}$ pour les fours de Curamba (Vetter et al., 2008), ce qui est suffissant pour fondre du plomb $\left(\sim 300^{\circ} \mathrm{C}\right)$. Cependant, des températures plus élevées sont nécessaires pour fondre l'argent $\left(960^{\circ} \mathrm{C}\right)$, I'or $\left(1063^{\circ} \mathrm{C}\right)$, ou le cuivre $\left(1084^{\circ} \mathrm{C}\right)$, alors que les alliages cuivre-argent ou cuivre-or demandent des températures de $780^{\circ}$ à $880^{\circ} \mathrm{C}$. Seuls quelques objets précolombiens en plomb ont été décrits (Petersen, 2010 [1970]; Vetter \& Villacorta, 2001; Vetter, 2004; Velarde, 2010) et il y a trés peu de références de sites précolombiens au Pérou comportant des preuves de fonderie d'argent (Wells, 1940; Howe \& Petersen, 1994).

Mots clés : plomb, fours, métallurgie, Inductively Coupled Plasma

\section{Ancient lead smelting at the Inca site of Curamba, Departament of Apurímac, Perú}

\begin{abstract}
Since 16th century, the Inca site of Curamba, Department of Apurímac, in southern Peru, has been interpreted as a metallurgical center for processing silver ore. However, there was no physical evidence such as fragments, or other indicators of the use of huayras or tocochimbos, which were structures traditionally used for pre-contact silver smelting in the Andes. Geochemical analyses (Inductively Coupled Plasma) of scoria excavated from the smelter pits at Curamba indicate low silver $(<0,3$ to 0,4 ppm), gold (<2 ppm), and copper (18 to $31 \mathrm{ppm}$ ) content whereas lead (155 to $234 \mathrm{ppm}$ ) and zinc (125 to $259 \mathrm{ppm}$ ) content were high. A lead-zinc signature is also indicated by the presence of arsenic, barium, iron, manganese, and vanadium. The bedrock geology at Curamba is comprised of south-dipping, fine-grained Mesozoic limestone of the regionally extensive Pucará Group. Lead-zinc occurrences are associated with the Mitu Formation, at the base of the Pucará, which crops out 1 $\mathrm{km}$ north of Curamba; however, no ore minerals such as galena [PbS] or sphalerite $[(\mathrm{Zn}, \mathrm{Fe}) \mathrm{S}]$ have been found at the site. On the basis of Mössbauer spectroscopy, a maximum temperature of $800 \pm$ $50{ }^{\circ} \mathrm{C}$ was inferred for the site (Vetter et al., 2008). This temperature is more than adequate to smelt lead $\left(\sim 300{ }^{\circ} \mathrm{C}\right)$; however, higher temperatures are required to smelt silver $\left(960{ }^{\circ} \mathrm{C}\right)$, gold $\left(1,063^{\circ} \mathrm{C}\right)$, or copper $\left(1,084^{\circ} \mathrm{C}\right)$ and copper-silver or copper-gold alloys require temperatures of $780^{\circ}$ to $880^{\circ} \mathrm{C}$, respectively. Only a few pre-contact lead artifacts have been described (Petersen, 2010 [1970]; Vetter \& Villacorta, 2001; Vetter, 2004; Velarde, 2010) and thus far there are few references to lead smelting sites in ancient Peru (Wells, 1940; Howe \& Petersen, 1994).
\end{abstract}

Key words: lead, ovens, metallurgy, Inductively Coupled Plasma 


\section{INTRODUCCIÓN}

Las ruinas del sitio de fundición Curamba se encuentran en el departamento de Apurímac, en la sierra sur del Perú, y están aproximadamente a medio camino entre Andahuaylas y Abancay, a una altura de 3700 msnm. Desde el siglo XVI, los arqueólogos, exploradores y geólogos han planteado que Curamba es un antiguo centro metalúrgico en el que se procesaban menas de plata. Esta interpretación se basó en la producción a gran escala de oro, plata y cobre por los antiguos peruanos; además en la ubicación del sitio en una colina para aprovechar los vientos para la fundición y en la presencia de más de 40 depresiones que fueron interpretadas como hornos.

Sin embargo, ninguna de las descripciones del sitio menciona la presencia de restos de huayras o tocochimbos', ni el hallazgo de minerales de plata en el sitio. Son escasas las investigaciones geoarqueológicas relacionadas con las actividades metalúrgicas en los Andes, y a pesar del papel legendario de Curamba en la metalurgia prehispánica, no hay información sobre los metales producidos en este sitio.

\section{ESTUDIOS PREVIOS EN EL SITIO DE CURAMBA}

Curamba fue descrito por primera vez en 1534 como un lugar utilizado por Pizarro y sus soldados durante su viaje a Cusco (Sancho de la Hoz, 1968 [1534]). El sitio está dominado por una plataforma ceremonial de $7 \mathrm{~m}$ de alto y $25 \mathrm{~m}$ de largo. Cerca de dicha plataforma se encuentran los hornos, distribuidos en tres andenes artificiales de aproximadamente $5 \mathrm{~m}$ de ancho y 1,6 $\mathrm{m}$ de alto. Desde 1534, el sitio ha sido visitado y definido como un centro metalúrgico, siendo las descripciones más detalladas las de Wiener (1993 [1880]), Olaechea (1901), Petersen (2010 [1970]), Lechtman (1976) y Del Mar (2006). Una investigación de campo hecha por Vetter et al. (2008) proporcionó datos analíticos sobre la edad del sitio y las temperaturas de los hornos; sin embargo, no aclaró de manera concluyente cuáles fueron los minerales y metales que se fundieron allí.

Se cree que durante la época prehispánica se trasladaron plateros del valle de Chimor de la costa norte hacia Curamba, y es posible que de Curamba fueran llevados a Cusco. Según Disselhoff (1978: 142; tomado de Espinoza, 1983: 38), este traslado se produjo2, pero como bien menciona Espinoza, aún no se ha encontrado documentación colonial que lo confirme. Hay que recordar que los incas movilizaron a grupos de artesanos de sus lugares de origen hacia el Cusco, para suplir algunas de sus necesidades. Las evidencias arqueológicas subrayan la gran destreza de los orfebres de la costa norte, por lo que resulta muy probable que ellos, al igual que los de la costa central, hayan sido trasladados al Cusco.

1 Estructuras tradicionales de arcilla o piedra asociadas con la fundición de plata antes del contacto europeo en los Andes.

2 Disselhoff no menciona si pasaron primero por Curamba y luego siguieron hacia Cusco. 
En todo caso, la información disponible permite inferir que, por lo menos, se llevó a cabo el traslado desde el valle de Chimor hacia Curamba. En efecto, en su recorrido por la sierra sur, Pedro Pizarro pasó por Curamba, donde se encontró con un grupo de personas que afirmaban dirigirse a Trujillo llevando unos tablones de plata para elaborar «una casa para su ydolo, que se llamaua Achimo» (Pizarro, 1978 [1571]: 80-81). Hay que tomar en cuenta que el área en donde se produjo el encuentro «estava disierto por averse huydo toda la gente dél» (Pizarro, 1978 [1571]: 80-81). Es decir, ya no existía el control estatal incaico que obligaba a estos probables especialistas norteños a permanecer en el área, como sí ocurrió en otras regiones ya que durante el periodo de la conquista española diversos grupos de mitmas optaron por retornar a sus tierras de origen - tal pudo ser el caso de los indios encontrados por Pedro Pizarro en Curamba-. Mientras tanto otros decidieron permanecer en los lugares donde habían sido trasladados por los incas, como el caso de los mitmas yungas plateros de la costa central.

Es posible que los indios que menciona Pedro Pizarro fuesen mitmas plateros del valle de Chimor que estaban de regreso a Trujillo, su lugar de origen, desde Curamba.

Otro dato que apoya esta hipótesis es la cita de un documento que trata de los títulos de las tierras de Coramba y Pecoy en el valle de Xaxahuana, provincia de Abancay, que Mateo Jiménez de Quesada compuso en el año de 1647 con el capitán don Fernando de Castilla Altamirano:

«... (fol. 1) las tierras de Matheo Ximenes $q^{\prime}$ estan en este balle en el aciento de pecoy y otros nombres que lindan por abaxo con el rrio de Piturayba y por un lado con las tierras de los yndios de Coramba y por arriba con tierras de los indios plateros hasta en derecho de un corral de cabras antiguo donde esta puesto un moxon de una crus... (fol. 5) y allo en ellas tres fanegas y media e puso por moxones y linderos por la parte de abaxo el rrio de Pitoray y por un lado las tierras de los yndios tomebambas y curambas, y por la parte de arriba tierras de los yndios yungas plateros de la ciudad del Cusco...» (AGN, 1647, Legajo 7, 145).

\section{CENTROS METALÚRGICOS EN EL PERÚ}

En la literatura arqueológica andina se ha mencionado muchas veces como centros metalúrgicos a Curamba, ubicado en la sierra sur, y a un área de quema cerca de la ciudadela Tschudi, en Chan Chan, costa norte. Esta última zona de quema abarca un área de 25 x 70 metros y fue interpretada como un centro metalúrgico chimú (1100-1450 d. C.) a causa de la presencia de artefactos de plata y escoria (Squier, 1877). Esta interpretación se mantuvo en la literatura (Pfordte, 1893; Caley \& Easby, 1959; Petersen, 2010 [1970]; Samamé Boggio, 1979) hasta que los estudios geoquímicos indicaron que la supuesta escoria era en realidad adobe muy quemado, sin evidencias de relación con la metalurgia. Hasta ahora el uso original de esa zona de quema es desconocido (Lechtman \& Moseley, 1975). Un muestreo geoquímico confirmó una ley muy baja de plata, cobre y oro en las muestras del 
suelo; sin embargo, el alto contenido de calcio y fósforo en las muestras de la zona quemada llevó al hallazgo de un fragmento calcinado de un cráneo humano, lo que corroboró la afirmación del coronel La Rosa en el sentido que el área de quema de la ciudadela Tschudi había sido utilizada para la cremación de seres humanos (La Rosa en Squier, 1877: 141; Brooks et al., 2008).

Además de compilar información de investigaciones y mapeo más tempranos en Curamba, Vetter et al. (2008) aplicaron espectroscopía Mössbauer y así lograron determinar que los hornos habían sido calentados a una temperatura máxima de $800 \pm 50{ }^{\circ} \mathrm{C}$. Los estudios de datación por termoluminiscencia (TL) indicaron que el sitio fue utilizado por última vez aproximadamente hacía 450 años. Sin embargo, los estudios de difracción de rayos $\mathrm{X}$ en las muestras de los hornos no tuvieron éxito en determinar cuáles fueron los minerales de mena. A pesar de sus contribuciones arqueométricas para comprender mejor el papel de Curamba en la metalurgia andina, Vetter et al. (2008) concluyeron que todavía queda allí «... algún metal que queda por identificar».

Para intentar definir qué metal fue fundido en Curamba, al igual que en el caso del área de quema cerca de la ciudadela Tschudi, se tomó muestras de piedras y escoria de los hornos de Curamba para el análisis de Plasma Acoplado Inductivamente (ICP, por sus siglas en inglés) (cuadro 1). Esto se hizo con la idea de definir el contenido metálico anómalo como un indicador del tipo de minerales fueron fundidos en Curamba.

\section{INTERPRETACIÓN}

A lo largo del territorio del Perú, y específicamente en Apurímac, se localizan ocurrencias de cobre, oro, plata y plomo-zinc (Ministry of Energy and Mines, 1999: 14, 13, 16 y 15 respectivamente; Ministerio de Energía y Minas, 2000: 15). Por su parte, Petersen (2010 [1970]: 48) indica que «a corta distancia [de Curamba] se ha encontrado siete pozos de mina derrumbados» pero en la descripción falta el rumbo y la distancia exacta. Estas ocurrencias e indicadores de minería se juntaron con estudios arqueológicos, informes geológicos y mineros, así como los resultados de ICP, para interpretar qué metal se fundió en Curamba.

\section{1. Oro}

Aunque hay numerosas ocurrencias de oro en Apurímac (Ministry of Energy and Mines, 1999: Ministerio de Energía y Minas, 2000: 15), los bajos niveles de oro en las muestras (cuadro 1), la ausencia de referencias arqueológicas al procesamiento del oro en el sitio, la distancia de los aluviones auríferos, y la ausencia de quimbaletes o molinetes (rocas que pesan 1 a 2 toneladas que son utilizadas para pulverizar la mena de oro; Petersen, 2010 [1970]: 75, 83; Cánepa, 2005), así como de residuos de la molienda del mineral de la veta de oro en el sitio, indican que este metal no fue procesado en Curamba. 
Cuadro 1 - Análisis ICP de Curamba, Apurímac, Perú*

\begin{tabular}{|c|c|c|c|c|c|c|c|c|}
\hline & BP1101 & BP1102 & BP1103a & BP1103b & BP1104 & BP1105 & BP1106a & BP1106b \\
\hline Ag & $<0,3$ & $<0,3$ & 0,4 & 0,4 & $<0,3$ & 0,4 & 0,4 & $<0,3$ \\
\hline Al & 34600 & 1230 & 30200 & 43900 & 1440 & 47300 & 35600 & 53500 \\
\hline As & 40 & 9 & 32 & 45 & 4 & 69 & 53 & 74 \\
\hline $\mathrm{Au}$ & $<2$ & $<2$ & $<2$ & $<2$ & $<2$ & $<2$ & $<2$ & $<2$ \\
\hline B & $<20$ & $<20$ & $<20$ & $<20$ & $<20$ & $<20$ & $<20$ & $<20$ \\
\hline Ba & 117 & 7 & 136 & 199 & 10 & 198 & 190 & 235 \\
\hline $\mathbf{B i}$ & $<3$ & $<3$ & $<3$ & $<3$ & $<3$ & $<3$ & $<3$ & $<3$ \\
\hline $\mathrm{Ca}$ & 5580 & 160000 & 105000 & 9720 & 350000 & 15700 & 27900 & 14400 \\
\hline $\mathrm{Cd}$ & $<0,5$ & 0,6 & 0,5 & $<0,5$ & $<0,5$ & 0,6 & 0,5 & 0,8 \\
\hline Co & 11 & $<1$ & 11 & 17 & $<1$ & 16 & 16 & 21 \\
\hline $\mathrm{Cr}$ & 29 & 5 & 32 & 45 & 4 & 42 & 44 & 57 \\
\hline $\mathrm{Cu}$ & 21 & 6 & 18 & 31 & 3 & 24 & 25 & 25 \\
\hline $\mathrm{Fe}$ & 31200 & 8160 & 29600 & 43500 & 2220 & 46100 & 39600 & 52700 \\
\hline Ga & $<10$ & $<10$ & $<10$ & $<10$ & $<10$ & $<10$ & $<10$ & $<10$ \\
\hline $\mathrm{Hg}$ & $<1$ & $<1$ & $<1$ & $<1$ & $<1$ & $<1$ & $<1$ & $<1$ \\
\hline K & 3040 & 1170 & 4990 & 5100 & 707 & 5730 & 4710 & 6920 \\
\hline La & 18 & 3 & 16 & 25 & 2 & 28 & 21 & 29 \\
\hline Mg & 2480 & 1400 & 5360 & 9280 & 3070 & 4920 & 8020 & 6370 \\
\hline Mn & 548 & 62 & 597 & 1070 & 22 & 961 & 824 & 1150 \\
\hline Mo & $<1$ & $<1$ & $<1$ & $<1$ & $<1$ & $<1$ & $<1$ & $<1$ \\
\hline $\mathrm{Na}$ & 515 & 176 & 368 & 458 & 198 & 535 & 276 & 483 \\
\hline $\mathrm{Ni}$ & 26 & 5 & 27 & 41 & 3 & 36 & 39 & 53 \\
\hline $\mathbf{P}$ & 3440 & 690 & 3050 & 2750 & 1370 & 3210 & 2980 & 4250 \\
\hline $\mathbf{P b}$ & 158 & 15 & 135 & 177 & 14 & 231 & 155 & 234 \\
\hline $\mathbf{S}$ & $<500$ & $<500$ & $<500$ & $<500$ & $<500$ & $<500$ & $<500$ & $<500$ \\
\hline Sb & $<3$ & $<3$ & $<3$ & $<3$ & $<3$ & $<3$ & $<3$ & $<3$ \\
\hline Sc & 6 & $<5$ & $<5$ & 8 & $<5$ & 9 & 7 & 11 \\
\hline Se & $<5$ & $<5$ & $<5$ & $<5$ & $<5$ & $<5$ & $<5$ & $<5$ \\
\hline $\mathrm{Sr}$ & 33 & 140 & 168 & 34 & 444 & 56 & 60 & 61 \\
\hline Th & $<3$ & $<3$ & $<3$ & $<3$ & $<3$ & $<3$ & $<3$ & $<3$ \\
\hline $\mathrm{Ti}$ & 496 & $<100$ & 445 & 711 & $<100$ & 852 & 656 & 828 \\
\hline $\mathrm{Tl}$ & $<5$ & $<5$ & $<5$ & $<5$ & $<5$ & $<5$ & $<5$ & $<5$ \\
\hline U & $<8$ & $<8$ & $<8$ & $<8$ & $<8$ & $<8$ & $<8$ & $<8$ \\
\hline V & 36 & 5 & 40 & 62 & 6 & 78 & 63 & 68 \\
\hline W & $<2$ & $<2$ & $<2$ & $<2$ & $<2$ & $<2$ & $<2$ & $<2$ \\
\hline $\mathrm{Zn}$ & 125 & 24 & 132 & 176 & 4 & 214 & 167 & 259 \\
\hline
\end{tabular}


* Resolución Directoral Nacional n. ${ }^{\circ}$ 1358/INCBP1101: rojo litología incierto, de un horno/hoyo quemado, 50-60 cm de ancho, muestra alzada a mano, con fragmentos de cuarzo, otros hoyos, poco profundo, andén 1

BP1102: caliza, gris oscuro, grano fino, vetillas de calcita, superficie rojo, sin quemar, muestra sesgada, posiblemente la pared del hoyo o quizás el «domo», en realidad, solamente una depresión, bien organizado, como una fogata, con rocas organizadas, tamaño cm (Mesozoico, grupo Pucará) (Marocco, 1975)

BP1103: hoyo, 1,8 m de longitud, $40 \mathrm{~cm}$ de ancho, zona quemada de $10 \mathrm{~cm}$ con algo negro, andén 2; a) polvo rojo/negro con fragmentos, ilitología?; b) iroca quemada?, iterrones?

BP1104: caliza, gris oscuro, grano fino, muestra alzada a mano, superficie rojo (Mesozoico, grupo Pucará) (Marocco, 1975)

BP1105: negro, suelo oxidado, escoria

BP1106: a) rojo/negro, desechos con cenizas del hoyo; b) rojo/negro, sustancia duro del hoyo

Inductively Coupled Plasma (ICP) análisis, en partes por millón (ppm), American Assay Laboratories, Sparks, NV.

\section{2. Cobre}

En Apurímac hay ocurrencias de cobre (Ministry of Energy and Mines, 1999: 14). Sin embargo, los bajos niveles de cobre en la escoria (cuadro 1), la ausencia de referencias arqueológicas al procesamiento del cobre en el sitio, la ausencia de minerales de mena de cobre y la ausencia en los hornos de manchas verdes típicas del cobre indican también que no fue procesado en Curamba.

\section{3. Plata}

Existen numerosas ocurrencias de plata en Apurímac (Ministry of Energy and Mines, 1999: 16); sin embargo, la ausencia de fragmentos de huayras o tocochimbos en el sitio indica que en Curamba no se fundió la galena argentífera, un mineral de plata común usado en los antiguos Andes. Las huayras y los tocochimbos son estructuras de piedra o arcilla que miden cerca de $1 \mathrm{~m}$ de altura y están asociados tradicionalmente con la antigua fundición andina de plata. La fundición inicial de la galena argentífera ocurre en la huayra, donde el azufre y algo de plomo son volatilizados por el fuego. Entonces, el rico metal de plata que queda es refinado una vez más en el tocochimbo para quitar el plomo y dejar solo la plata que se destinará a la orfebrería (Ahlfeld \& SchneiderScherbina, 1964; Petersen, 2010 [1970]; Van Buren \& Mills, 2005). Otros datos importantes incluyen el bajo contenido de plata en las escorias (cuadro 1) y el análisis de TL que indica que una temperatura máxima de $800 \pm 50{ }^{\circ} \mathrm{C}$ fue alcanzada en los hornos de Curamba, mientras que una temperatura de $960{ }^{\circ} \mathrm{C}$ es requerida para fundir la plata. 


\section{4. Plomo-zinc}

De los metales discutidos, el contenido de plomo-zinc de las muestras de Curamba es el más alto (135-234 ppm Pb y 125-259 ppm Zn) (cuadro 1) y esto es un elemento contundente para afirmar que Curamba fue un antiguo sitio de fundición de plomo. Los valores de otros metales anómalos que también pueden estar asociados con depósitos de plomo-zinc —como arsénico, bario, cromo, hierro, manganeso y vanadio- son también altos. Estos metales pueden asociarse con galena, el mineral común de plomo, o esfalerita, el mineral común de zinc (Deer et al., 1966: 461; Ali Mattash, 2008).

Existen aproximadamente diez ocurrencias de plomo-zinc en Apurímac, la mayoría en el noreste del departamento (Ministry of Energy and Mines, 1999: 15). Estos se encuentran en, o cerca de, los afloramientos de arcillas rojas, conglomerados y rocas volcánicas (rumbo noroeste), de la Formación Mitu (Mesozoico), en la base del grupo Pucará (Marocco, 1975). En el Perú, la Formación Mitu es el anfitrión regional de las ocurrencias de plomo-zinc (Richard Petersen, geólogo asesor, comunicación personal, 2011) y hay afloramientos de esta formación a solo $1 \mathrm{~km}$ al norte de Curamba (Marocco, 1975). También se encuentran afloramientos de la Formación Mitu en la cuenca Pucará, y las ocurrencias de plomo-zinc están normalmente asociadas con zonas de cizallamiento (Rosas et al., 2007). Por comparación, rocas sedimentarias con litología muy semejante a las de Pucará-Mitu tienen normalmente solo 7-9 ppm Pb y 16-20 ppm Zn (Turekian \& Wedepohl, 1961).

En Curamba, los andenes donde se encuentran los hornos tienen un relleno de arcilla densa y roja que es claramente diferente de la piedra caliza de color gris que se encuentra cerca del sitio. El material ha sido descrito como foráneo (Lechtman, 1976) y actualmente este relleno es interpretado como correlativo a las arcillas rojas de la Formación Mitu. Esto indica que las rocas de dicha formación fueron conocidas, transportadas y utilizadas como relleno para los andenes en Curamba.

Los hornos de Curamba miden aproximadamente $40 \mathrm{~cm}$ de ancho y $1,8 \mathrm{~m}$ de largo, están compuestos de bloques de caliza grises y de grano fino del grupo Pucará. La geometría de los hornos es aproximadamente igual al tamaño y forma de los hornos de plomo de la época romana en el Reino Unido (Craddock, 1995: 206). El combustible utilizado en Curamba fue carbón de leña y posiblemente también se utilizó maíz. La espectroscopía de Mössbauer estableció una temperatura máxima de $800 \pm 50{ }^{\circ} \mathrm{C}$ para los hornos de Curamba (Vetter et al., 2008) y esta temperatura habría sido más que adecuada para fundir plomo $\left(\sim 300^{\circ} \mathrm{C}\right)$.

\section{DISCUSIÓN}

La galena es el mineral común de plomo en los Andes y los mineros peruanos establecen diferencias entre dos tipos de galena: 1) sorojche es la galena de cristales pequeños y argentíferos. Por ejemplo, una muestra de sorojche de Porco, 
Bolivia, tenía 3,250 ppm Ag (Brooks \& Van Buren, 2007), y por el contrario, 2) la galena macrocristalina es pobre en plata (Petersen, 2010 [1970]: 6). El plomo nativo se encuentra raramente, y por lo tanto no habría sido una fuente del metal. Petersen (2010 [1970]: 30) afirma que se encontraron cuentas (mm) de plomo nativo en placeres de oro-platino en el noroeste de Choco, Colombia (Petersen, 2010 [1970]: 30). La galena argentífera o sorojche fue mucho más importante como parte de la producción de plata en los antiguos Andes; sin embargo, también se utilizó plata nativa (Van Buren \& Mills, 2005).

Baessler (1906) postuló que en el Perú solo se fundió plomo después de la llegada de los europeos, pero Petersen (2010 [1970]: 30) tiene descripciones de varios artefactos prehispánicos, algunos de la cultura Moche (100 a. C.-700 d. C.), lo que confirmaría el procesamiento de la mena de plomo por la minería prehispánica. Además se conocen objetos de la costa central elaborados con plomo (Vetter \& Villacorta, 2001; Vetter, 2004); así también en el valle del Mantaro se han encontrado evidencias de fundición de plomo, desde escorias y lingotes hasta piezas elaboradas con ese metal de la época Wanka (Howe \& Petersen 1994). En el Perú existen artefactos precolombinos de plomo que se encuentran en colecciones de museos como el Etnológico de Berlín en Alemania o el Museo Arqueológico Brüning en Lambayeque, Perú; así también en el Museo Amano y el Museo Larco en Lima (Petersen, 2010 [1970]: 31); y el Museo de América en Madrid (Cuesta, 1980: 210). En el catálogo en línea del Museo Larco se pueden apreciar 17 artefactos de plomo precolombino descritos como lingotes, pesas, entre otros (Museo Larco, s. f.).

Petersen (2010 [1970]: 31) realizó el análisis de un vaso de plomo de La Ventana, un sitio arqueológico chimú (1100-1450 d. C.) localizado cerca de Lambayeque, en el norte del Perú. Este análisis reveló 99 \% de plomo y $<0,01 \%$ de plata y es un indicador de dos hechos importantes: 1) la producción de plomo como un material primario para el uso industrial de la galena no argentífera, 2) el plomo no fue un subproducto de la producción de plata argentífera. En 2009, un pedazo doblado de plomo, que medía 2 × $3 \mathrm{~cm}$ y $4 \mathrm{~mm}$ de grosor, fue identificado como hallazgo arqueológico en El Purgatorio, cerca de Casma (Melissa Vogel, comunicación personal, 11 de agosto de 2011); además, hay ocurrencias de plomo-zinc cerca de allí (Sánchez et al., 1995: 211). En Colombia y Ecuador también se conocen artefactos de plomo de la época anterior al contacto con Europa.

En la arqueología del Viejo Mundo, las minas de galena cerca de Laurion, en el sur de Grecia, proporcionaron galena argentífera (Healy, 1978: 157) que fue refinada para proporcionar la plata que se utilizaría para financiar la derrota de Pérsico en 478-479 a. C. Ocurrencias de galena no argentífera también habrían sido explotadas para proporcionar plomo que fue utilizado en el Partenón para suavizar los efectos de los sismos en la región (Hadingham, 2008). De manera semejante al caso de la minería de plomo en los Andes prehispánicos, estos hechos indican conocimiento de la diferencia entre galena argentífera y galena no argentífera, así como la antigua explotación griega de galena argentífera por su contenido de plata y la minería de galena no argentífera para el uso industrial. 


\section{CONCLUSIÓN}

A pesar de la ausencia de huayras y tocochimbos y de minerales de mena de plata en el sitio, Curamba fue interpretado como un lugar de fundición de plata utilizado desde el siglo XVI, antes de la llegada de los españoles. La integración de las pruebas físicas, las investigaciones arqueológicas y geológicas, la espectroscopía de Mössbauer, la datación por termoluminiscencia, y la geoquímica de las escorias de los hornos de fundición son consecuentes con la interpretación de Curamba como un centro de hornos de fundición para el procesamiento de la galena no argentífera. La evidencia de minas derrumbadas y la proximidad de rocas con menas de plomo-zinc de la Formación Mitu conforman también un aporte importante y apoyan esta conclusión. La presencia —aunque no abundante- de artefactos de plomo prehispánicos en las colecciones de los museos del Perú, y otras extranjeras, también indica que el plomo no argentífero fue fundido para el uso industrial en esa época.

Asimismo, los documentos coloniales señalan que los plateros (ARC, 1664, Corregimiento, Prot. Notarial 202, folios 1226-1227v. Escribano Lorenzo Meza Andueza) no solo trabajaban con plata, también lo hacían con otros metales como el oro y el cobre. Aunque los documentos mencionan que eran yungas plateros, posiblemente los de Curamba elaboraban piezas de plomo.

\section{Referencias citadas}

\section{Fuentes Primarias}

Archivo General de la Nación, Lima (AGN)

Archivo Regional de Cusco (ARC)

\section{Fuentes Secundarias}

AHLFELD, F. \& SCHNEIDER-SCHERBINA, A., 1964 - Los yacimientos minerales y de hidrocarburos de Bolivia, 388 pp.; La Paz: Ministerio de Minas y Petróleo, Departamento Nacional de Geología.

ALI MATTASH, M., 2008 - Geology and geochemistry of the sedimentary hosted leadzinc-vanadium-barite prospects in Yemen (abstract). International Geological Congress, Oslo, Norway, August 6-14. Disponible en: http://www/cprm.gov. $\mathrm{br} / 33 \mathrm{IGC} / 1287218 . \mathrm{html}$

BAESSLER, A., 1906 - Altperuanische Metallgerate, 225 pp.; Berlín: Druck und Verlagsanstalt.

BROOKS, W. E. \& VAN BUREN, M., 2007 - Dirty galena at Porco, Bolivia. Ceological Society of America Abstracts with Programs, 39 (6): 549.

BROOKS, W. E., GÁLVEZ MORA, C., JACKSON, J. C., MCGEEHIN, J. P. \& HOOD, D. G., 2008 - Coal and cremation at the Tschudi burn, Chan Chan, northern Peru. Archaeometry, 50 (3): 495-515. 
Antigua fundición de plomo en el sitio inca de Curamba, departamento de Apurímac, Perú

CALEY, E. R. \& EASBY, D. T., 1959 - The smelting of sulfide ores of copper in preconquest Peru. American Antiquity, 25 (1): 59-65.

CÁNEPA, C., 2005 - Minería a pequeña escala en la costa sur media del Perú, 85 pp.; Lima: Instituto Geológico Minero y Metalúrgico.

CRADDOCK, P., 1995 - Early metal mining and production, 363 pp.; Washington, D. C.: Smithsonian Institution Press.

CUESTA, M., 1980 - Arqueología Andina: Perú, 444 pp.; Madrid: Museo de América.

DEER, W. A., HOWIE, R. A. \& ZUSSMAN, J., 1966 - An introduction to the rock-forming minerals, 696 pp.; Londres: Longmans.

DEL MAR, I., 2006 - Curamba, centro de producción metalúrgica de época Inka. Arqueología y Sociedad, 17: 293-312.

ESPINOZA SORIANO, W., 1983 - Los mitmas plateros de Ishma en el país de los Ayamarca siglos XV-XIX. Boletín de Lima, 30 (5): 38-52; Lima.

HADINGHAM, E., 2008 - Unlocking mysteries of the Parthenon. Smithsonian Magazine, 38 (11): 36-43. Disponible en: http://www.smithsonianmag.com/history-archaeology/ Unlocking-Mysteries-of-the-Parthenon.html

HEALY, J. F., 1978 - Mining and Metallurgy in the Greek and Roman World, 316 pp.; London: Thames and Hudson.

HOWE, E. \& PETERSEN, U., 1994 - Silver and lead in the Late Prehistory of the Mantaro Valley, Peru. In: Archaeometry of Pre-Columbian sites and artifacts. Proceedings of a Symposium organized by the UCLA Institute of Archaeology and the Getty Conservation Institute, Los Angeles, California, March 23-27, 1992 (D. Scott \& P. Meyers, eds.): 183-198; Los Ángeles: The Getty Conservation Institute.

LECHTMAN, H., 1976 - A metallurgical site survey in the Peruvian Andes. Journal of Field Archaeology, 3 (1): 1-42.

LECHTMAN, H. N. \& MOSELEY, M., 1975 - The scoria at Chan-Chan, non-metallurgical Deposits. Nawpa Pacha, 10 (12): 135-185.

MAROCCO, R., 1975 - Geología de los cuadrángulos de Andahuaylas, Abancay y Cotabambas, 51 pp.; Lima: Ministerio de Energía y Minas, Instituto de Geología y Minería.

MINISTERIO DE ENERGÍA Y MINAS, 2000 - Atlas de la pequeña minería en el Perú 2000; Lima: Ministerio de Energía y Minas, Dirección General de Minería.

MINISTRY OF ENERGY AND MINES, 1999 - Atlas Mining and energy in Peru; Lima: Ministry of Energy and Mines.

MUSEO LARCO, s. f. - Tesoros del antiguo Perú, plomo; Lima: Museo Larco. Disponible en: http://www.museolarco.org/

OLAECHEA, T., 1901 - Apuntes sobre el Castillo y fundición de Curamba. Anales de Construcción Civiles, Minas e Industrias del Perú, 1 (2): 1-21; Lima: Escuela de Ingenieros de Lima.

PETERSEN, G., 2010 [1970] - Mining and metallurgy in ancient Perú, 97 pp.; Boulder, CO: Geological Society of America.

PFORDTE, O. F., 1893 - Ancient method of silver-lead smelting in Perú. Transactions of the American Institute of Mining Engineers, 21: 25-30.

PIZARRO, P., 1978 [1571] - Relación del Descubrimiento y Conquista de los Reinos del Perú, 376 pp.; Lima: PUCP.

ROSAS, S., FONTBOTÉ, L. \& TANKARD, A., 2007 - Tectonic evolution and paleogeography of the Mesozoic Pucará Basin, central Peru. Journal of South American Earth Sciences, 24: 1-24, doi:10.1016/j.jsames.2007.03002 
SAMAMÉ, M., 1979 - El Perú minero, Tomo I; Lima: Instituto Científico y Tecnológico Minero.

SÁNCHEZ, A., MOLINA, O. \& GUTIÉRREZ, R., 1995 - Geología de los cuadrángulos de Chimbote, Casma y Culebras: hojas 19-f, 19-g y 20-g, 263 pp.; Lima: Instituto Geológico Minero y Metalúrgico.

SANCHO DE LA HOZ, P., 1968 [1534] - Relación para su Majestad de Pedro Sancho de la Hoz, 1534. In: El Perú a través de los siglos: 275-343; Lima: Universidad Nacional Mayor de San Marcos, Editores Técnicos Asociados.

SQUIER, E. G., 1877 - Peru, Incidents of travel and exploration in the land of the Incas, 599 pp.; Nueva York: Harper and Brothers.

TUREKIAN, K. K. \& WEDEPOHL, K. H., 1961 - Distribution of the elements in some major units of the Earth's crust, Geological Society of America Bulletin, 72: 175-192.

VAN BUREN, M. \& MILLS, B. H., 2005 - Huayrachinas and tocochimbos, traditional smelting technology of the southern Andes. Latin American Antiquity, 16 (1): 3-25.

VELARDE, M. I., 2010 - Las evidencias de metales en Cashamarca. In: Cashamarca: Su ubicación dentro del proceso histórico del Antiguo Perú (M. Calderón Lazo, ed.): 79-95; Lima: Cemento Andino.

VETTER, L., 2004 - Las piezas de metal del Museo de Sitio Arturo Jiménez Borja-Puruchuco. In: Puruchuco y la sociedad de Lima: un homenaje a Arturo Jiménez Borja (L. F. Villacorta, L. Vetter \& C. Ausejo, eds.): 119-139; Lima: Concytec, Compañía de Minas Buenaventura S. A. A.

VETTER, L. \& VILLACORTA L. F., 2001 - La arqueometalurgia de la costa central del Perú: Una perspectiva desde la colección del Museo de Sitio Arturo Jiménez BorjaPuruchuco. Baessler Archiv, 49: 193-210.

VETTER, L., PETRICK, S., HUAYPAR, Y. \& MACKAY FULLE, M., 2008 - Los hornos metalúrgicos del sitio Inca de Curamba, Perú. Bulletin de I'Institut Francais d'Etudes Andines, 37 (3): 451-475.

WELLS, R., 1940 - Una inspección superficial de sitios arqueológicos cerca de La Oroya, Perú. In: Actas y trabajos científicos del XXXII Congreso Internacional Americanistas, Tomo I: 347-354.

WIENER, C., 1993 [1880] - Perú y Bolivia, relato de viaje, 859 pp.; Lima: Instituto Francés de Estudios Andinos, Universidad Nacional Mayor de San Marcos. 